\title{
SOLVABILITY OF GENERAL BACKWARD STOCHASTIC VOLTERRA INTEGRAL EQUATIONS
}

\author{
Yufeng Shi And Tianxiao Wang
}

\begin{abstract}
In this paper we study the unique solvability of backward stochastic Volterra integral equations (BSVIEs in short), in terms of both the adapted M-solutions introduced in [19] and the adapted solutions via a new method. A general existence and uniqueness of adapted Msolutions is proved under non-Lipschitz conditions by virtue of a briefer argument than the ones in [13] and [19], which modifies and extends the results in [13] and [19] respectively. For the adapted solutions, the unique solvability of BSVIEs under more general stochastic non-Lipschitz conditions is shown, which improves and generalizes the results in [7], [14] and [15].
\end{abstract}

\section{Introduction}

Let $\left\{W_{t}\right\}_{t \in[0, T]}$ be a $d$-dimensional Wiener process defined on a complete probability space $(\Omega, \mathcal{F}, P)$ and $\mathbb{F} \equiv\left\{\mathcal{F}_{t}\right\}$ denote the natural filtration of $\left\{W_{t}\right\}$, such that $\mathcal{F}_{0}$ contains all $P$-null sets of $\mathcal{F}$. This paper is motivated by the recent works of Yong ([17], [19]), which studied an important extension of backward stochastic differential equations (BSDEs in short), i.e., backward stochastic Volterra integral equations (BSVIEs in short). The following wellknown nonlinear BSDEs

$$
Y(t)=\xi+\int_{t}^{T} g(s, Y(s), Z(s)) d s-\int_{t}^{T} Z(s) d W(s)
$$

Received August 12, 2011; Revised April 17, 2012.

2010 Mathematics Subject Classification. 60H10, 60H15, 60G55.

Key words and phrases. backward stochastic Volterra integral equations, adapted solutions, adapted M-solutions, non-Lipschitz conditions, stochastic Lipschitz coefficients.

Partially supported by National Natural Science Foundation of China (Grant 10771122) and (Grant 11071145), Natural Science Foundation of Shandong Province of China (Grant Y2006A08), Foundation for Innovative Research Groups of National Natural Science Foundation of China (Grant 10921101), National Basic Research Program of China (973 Program, No. 2007CB814900), Independent Innovation Foundation of Shandong University (Grant 2010JQ010), and Graduate Independent Innovation Foundation of Shandong University (GIIFSDU). 
initiated by Pardoux and Peng [11], have been studied extensively in the past two decades. The reader is referred to the books of Ma and Yong [8], Yong and Zhou [20] and the survey paper of El Karoui, Peng and Quenez [6] and so on for the detailed account of both theory and applications (especially in mathematical finance and stochastic controls) of BSDE (1.1). As a remarkable development of BSDEs, BSVIEs of the form

$$
Y(t)=\psi(t)+\int_{t}^{T} g(t, s, Y(s), Z(t, s), Z(s, t)) d s-\int_{t}^{T} Z(t, s) d W(s),
$$

were firstly introduced by Yong [17]. We refer the reader to [2], [13], [14], [17], [18] and [19] for both theory and applications (especially in dynamic risk measures and optimal controls) of BSVIEs. As to the adapted solution of (1.2) in $\mathcal{H}_{t}^{2}[0, T]$ in the case where $g$ is independent of the term $Z(s, t)$ or $\psi(t)=\xi$, the reader is referred to [1], [7], [15] and the references cited therein.

It will become more and more clear for BSVIEs to increasingly bring out incontestable significance in the theory and applications. However there are still many investigations to need in the fundamental theory of BSVIEs, such as unique solvability, comparison theorems and so on. In the existing results as in the papers mentioned above, the approaches are very complicated and intricate in form, even there are some gaps in their arguments.

The aim of this paper is to propose a new brief method to investigate the unique solvability of BSVIEs within a very general framework. Our results and arguments will enrich and perfect the foundational theory of BSVIEs. Inspired by the ideas in [5], firstly we will study the adapted M-solutions for (1.2) by virtue of a briefer argument than the ones in [19] (The adapted M-solution will be simplified as the M-solutions whenever it can be unambiguous in this paper). The reasons are at least twofold. On the one hand, in order to prove the unique existence of M-solutions for (1.2), the author had to make many preparations in the arguments in [19], such as the solvability of certain stochastic Fredholm integral equations (see Corollary 3.5 in [19]) and some complicated estimates of M-solutions for certain simple BSVIEs (see (3.29)-(3.31) in [19]). On the other hand, since BSVIEs do not have time-consistency (or semigroup property), and the process $Z$ has two time parameters, in order to make use of the induction method in the arguments for BSVIEs, the author had to get across four steps in [19] (see also [2] and [13]), which seems rather complicated and sophisticated. Therefore we intend to introduce a new convenient argument, that is, as the time interval $[0, T]$ is finite, we can make use of an equivalent norm in $\mathcal{H}^{2}[0, T]$ as follows:

$\|(y(\cdot), z(\cdot, \cdot))\|_{\mathcal{H}^{2}[0, T]}=\left[E \int_{0}^{T} e^{\beta A(t)}|y(t)|^{2} d t+E \int_{0}^{T} \int_{0}^{T} e^{\beta A(s)}|z(t, s)|^{2} d s d t\right]^{\frac{1}{2}}$,

where $\beta$ is a positive constant and $A(t)$ is assumed to be bounded. Then we will be able to prove the results for M-solutions within the new norm by one step. This is our first contribution in this paper. 
No matter the adapted M-solutions in [2], [13], [17], [18] and [19], or the adapted solutions in [7] and [15], the authors needed at least one of the following assumptions: 1) $g$ is independent of $Z(s, t), 2)$ the terminal condition is $\mathcal{F}_{T^{-}}$ measurable random variable $\xi, 3)$ the Lipschitz condition is imposed, moreover the Lipschitz coefficient is deterministic, 4) the deterministic non-Lipschitz condition is satisfied. In the present paper, without the above assumptions we consider the general setting of BSVIEs in terms of the above two kinds of solutions, respectively.

The adapted solutions of $(1.2)$ in $\mathcal{H}^{2}[0, T]$ is not unique due to the appearance of $Z(s, t)$ in the equations, (see Example 1.1 in [19]). Consequently, when $g$ is independent of $Z(s, t)$, the authors obtained the existence and uniqueness of adapted solution of $(1.2)$ in $\mathcal{H}_{t}^{2}[0, T]$ under Lipschitz conditions in [14]. In this paper we will develop the investigation on adapted solutions under stochastic non-Lipschitz conditions by adopting a new approach, which considerably improves and extends the results in [7], [14] and [15]. This is another contribution in this paper.

Motivated by the key ideas in classical Lipschitz case, we will use the equivalent norm above to study the existence and uniqueness of M-solutions for (1.2) in $\mathcal{H}^{2}[0, T]$ under weaker conditions. Recently Ren [13] considered the unique solvability of M-solutions under non-Lipschitz condition by adopting the method proposed by Anh and Yong in [2]. However, there is one problem ought to be pointed out. Firstly it is obvious that the splitting procedure in p.7 in [13] is lack in generality. Because the hypothesis (H3) in [13] is unreasonable even in the simplest case, (see Example 1 in the present paper). In this paper, we will bridge this gap by specifying some new assumptions which are weaker and more natural than the ones in [13]. Furthermore, it is clear that one cannot use the approaches in [15] directly to treat the existence of M-solutions for $(1.2)$ in $\mathcal{H}^{2}[0, T]$ under non-Lipschitz conditions. In fact, although in the special case of $g$ being independent of $Z(s, t)$, one can use the similar procedure as in [15] to get the solvability of M-solutions in $\mathcal{H}^{2}[0, T]$, but, as to the general form of (1.2), the approaches in [15] (see also the existence on p. 10 in [13]) does not work well any more due to the appearance of $E \int_{t}^{T}|Z(s, t)|^{2} d s$, which is rather difficult to estimate excepting introducing certain Malliavin calculus, (see Section 4 in [13]). Therefore in order to overcome this difficulty, we should apply some new ideas rather than copy the ones in [15]. By the definition of adapted M-solution and stochastic Fubini theorem, we notice the following fact

$$
E \int_{0}^{T} \int_{t}^{T}|Z(s, t)|^{2} d s d t=E \int_{0}^{T} \int_{0}^{t}|Z(t, s)|^{2} d s d t \leq E \int_{0}^{T}|Y(t)|^{2} d t,
$$

which implies that one should use the norm

$$
\int_{u}^{T} e^{\beta A(t)} E|Y(t)|^{2} d t+E \int_{u}^{T} \int_{t}^{T} e^{\beta A(s)}|Z(t, s)|^{2} d s d t
$$


with a suitable adapted process $A, u \in[0, T],(Y, Z) \in \mathcal{H}^{2}[0, T]$, rather than the norm

$$
e^{\beta t} E|Y(t)|^{2}+E \int_{t}^{T} e^{\beta s}|Z(t, s)|^{2} d s
$$

in [15]. With this new norm, we also need to apply a new Jensen's inequality by virtue of the property of convex function, which is different from the corresponding skills in [15]. After these preparations we can obtain the unique existence of M-solutions for BSVIE (1.2) under non-Lipschitz condition, which modifies and generalizes the results in [2], [13], [17], [18] and [19]. Eventually we can claim that our methods here can also work well in more general cases, such as the infinite dimension cases in [2] and [13], and the case of BSVIEs with jumps in [13] and [15]. For simplicity of presentation in this paper, we omit the discussion for the BSVIEs with jumps.

After the completion of this manuscript, we became aware of a recent work of Wang and Zhang [16] where the solvability of M-solutions under non-Lipschitz case was discussed. Comparing with the results and arguments in [16], there are three points worthy to point out. Firstly our assumptions on the related coefficients are much weaker than theirs, see (H2) below. Actually we allow both the Lipschitz coefficients and the non-Lipschitz coefficients to be certain integral functions rather than a constant. Secondly our method is totally different from ones in [16]. Our arguments are much briefer than theirs. For example, they used Picard's iteration that seems complicated and intricate, to prove the existence result for M-solutions. In this paper we study the general non-Lipschitz cases in virtue of the results in Lipschitz cases. Our approaches circumvent many unnecessary procedures. Thirdly, our approaches do not involve Itô formula which was heavily depended on in [15] and [16] . It is believed that one important reason of the coefficients there being constants lies in not only the limitation of their approach, but also the dependence of Itô formula, see Lemma $3.1 \mathrm{in} \mathrm{[15].} \mathrm{Thus} \mathrm{this} \mathrm{is} \mathrm{another} \mathrm{reason} \mathrm{why} \mathrm{we} \mathrm{avoid} \mathrm{the} \mathrm{appearance}$ of Itô formula in our approach.

The paper is organized as follows. In Section 2, we give some preliminary results and notations which are needed in the following sections. An important estimate for M-solutions (or adapted solutions) is presented in Subsection 3.1. With this estimate, we give the existence and uniqueness result of Msolutions under Lipschitz conditions in Subsection 3.2. The case of adapted solutions is also treated. In Subsection 3.3, we consider the unique solvability of M-solutions and adapted solutions, respectively, under non-Lipschitz conditions. At last an example about M-solutions and adapted solutions under non-Lipschitz condition is presented in Section 4.

\section{Preliminaries}

In this section, we will make some preliminaries. In the following we denote

$$
\Delta^{c}[R, S] \doteq\left\{(t, s) \in[R, S]^{2} ; t \leq s\right\}, \quad \Delta^{c} \doteq \Delta^{c}[0, T],
$$


GENERAL BACKWARD STOCHASTIC VOLTERRA INTEGRAL EQUATIONS 1305

$$
\Delta[R, S] \doteq\left\{(t, s) \in[R, S]^{2} ; t>s\right\}, \quad \Delta \doteq \Delta[0, T], \quad R, S \in[0, T] .
$$

Let $\beta$ be a positive constant. $A(t)$ is a non-negative $\mathbb{F}$-adapted increasing process.

$$
\begin{gathered}
L_{\mathcal{F}_{T}}^{2}\left(R, S ; \mathbb{R}^{m}\right) \doteq\left\{X:[R, S] \times \Omega \rightarrow \mathbb{R}^{m} \mid X(\cdot) \text { is } \mathcal{F}_{T^{-}}\right. \text {-measurable process } \\
\left.\quad \text { such that } E \int_{R}^{S}|X(s)|^{2} d s<\infty\right\} . \\
L_{\mathcal{F}_{T}}^{2, \beta}\left(R, S ; \mathbb{R}^{m}\right) \doteq\left\{X:[R, S] \times \Omega \rightarrow \mathbb{R}^{m} \mid X(\cdot) \text { is } \mathcal{F}_{T^{-} \text {-measurable process }}\right. \\
\text { such that } \left.E e^{\beta A(S)} \int_{R}^{S}|X(s)|^{2} d s<\infty\right\} .
\end{gathered}
$$

From the above two definitions, we know $L_{\mathcal{F}_{T}}^{2, \beta}\left(R, S ; \mathbb{R}^{m}\right) \subset L_{\mathcal{F}_{T}}^{2}\left(R, S ; \mathbb{R}^{m}\right)$. We denote by

$$
\begin{aligned}
& \mathcal{H}^{2}[R, S]=L_{\mathbb{F}}^{2}\left(R, S ; \mathbb{R}^{m}\right) \times L^{2}\left(R, S ; L_{\mathbb{F}}^{2}\left(R, S ; \mathbb{R}^{m \times d}\right)\right), \\
& \mathcal{H}_{t}^{2}[R, S]=L_{\mathbb{F}}^{2}\left(R, S ; \mathbb{R}^{m}\right) \times L^{2}\left(R, S ; L_{\mathbb{F}}^{2}\left(t, S ; \mathbb{R}^{m \times d}\right)\right), \quad t \in[R, S],
\end{aligned}
$$

where

$$
\begin{aligned}
& L_{\mathbb{F}}^{2}\left(R, S ; \mathbb{R}^{m}\right) \doteq\left\{X:[R, S] \times \Omega \rightarrow \mathbb{R}^{m} \mid X(\cdot) \text { is } \mathbb{F}\right. \text {-adapted process such that } \\
& \left.\quad E \int_{R}^{S}|X(s)|^{2} d s<\infty\right\} \\
& L^{2}\left(R, S ; L_{\mathbb{F}}^{2}\left(R, S ; \mathbb{R}^{m \times d}\right)\right) \doteq\left\{Z:[R, S]^{2} \times \Omega \rightarrow \mathbb{R}^{m \times d} \mid Z(t, \cdot) \text { is } \mathbb{F}\right. \text {-adapted } \\
& \text { process, for almost all } \left.t \in[R, S], E \int_{R}^{S} \int_{R}^{S}|Z(t, s)|^{2} d s d t<\infty\right\}, \\
& L^{2}\left(R, S ; L_{\mathbb{F}}^{2}\left(t, S ; \mathbb{R}^{m \times d}\right)\right) \doteq\left\{Z: \Delta^{c}[R, S] \times \Omega \rightarrow \mathbb{R}^{m \times d} \mid Z(t, \cdot) \text { is } \mathbb{F}\right. \text {-adapted } \\
& \text { process, for almost all } \left.t \in[R, S], E \int_{R}^{S} \int_{t}^{S}|Z(t, s)|^{2} d s d t<\infty\right\} .
\end{aligned}
$$

Note that it is obvious that $L_{\mathbb{F}}^{2}\left(R, S ; \mathbb{R}^{m}\right) \subset L_{\mathcal{F}_{T}}^{2}\left(R, S ; \mathbb{R}^{m}\right)$. We also denote by

$$
\begin{aligned}
& \mathcal{H}^{2, \beta}[R, S] \doteq L_{\mathbb{F}}^{2, \beta}\left(R, S ; \mathbb{R}^{m}\right) \times L^{2, \beta}\left(R, S ; L_{\mathbb{F}}^{2}\left(R, S ; \mathbb{R}^{m}\right)\right), \\
& \mathcal{H}_{t}^{2, \beta}[R, S] \doteq L_{\mathbb{F}}^{2, \beta}\left(R, S ; \mathbb{R}^{m}\right) \times L^{2, \beta}\left(R, S ; L_{\mathbb{F}}^{2}\left(t, S ; \mathbb{R}^{m \times d}\right)\right), \quad t \in[R, S],
\end{aligned}
$$

where for example

$L_{\mathbb{F}}^{2, \beta}\left(R, S ; \mathbb{R}^{m}\right)=\left\{X:[R, S] \times \Omega \rightarrow \mathbb{R}^{m} \mid X(\cdot)\right.$ is $\mathbb{F}$-adapted process such that

$$
\left.E \int_{R}^{S} e^{\beta A(s)}|X(s)|^{2} d s<\infty\right\}
$$


Here we also have some relations between the spaces above, for example,

$$
L_{\mathcal{F}_{T}}^{2, \beta}\left(R, S ; \mathbb{R}^{m}\right) \subset L_{\mathcal{F}_{T}}^{2}\left(R, S ; \mathbb{R}^{m}\right), \quad L_{\mathbb{F}}^{2, \beta}\left(R, S ; \mathbb{R}^{m}\right) \subset L_{\mathbb{F}}^{2}\left(R, S ; \mathbb{R}^{m}\right) .
$$

Now we give two definitions needed in the sequel.

Definition 2.1. Let $S \in[0, T]$. A pair of $(Y(\cdot), Z(\cdot, \cdot)) \in \mathcal{H}^{2, \beta}[S, T]$ is called an adapted $M$-solution of BSVIE (1.2) on $[S, T]$, if (1.2) holds in the usual Itô's sense for almost all $t \in[S, T]$ and, in addition, for any $t \in[S, T]$,

$$
Y(t)=E^{\mathcal{F}_{S}} Y(t)+\int_{S}^{t} Z(t, s) d W(s) .
$$

Definition 2.2. A pair of $(Y(\cdot), Z(\cdot, \cdot)) \in \mathcal{H}_{t}^{2, \beta}[0, T]$ is called an adapted solution of the following simple BSVIE (2.3), if (2.3) holds in the usual Itô's sense for almost $t \in[0, T]$,

$$
Y(t)=\psi(t)+\int_{t}^{T} g(t, s, Y(s), Z(t, s)) d s-\int_{t}^{T} Z(t, s) d W(s) .
$$

In [19], Yong introduced the notion of adapted M-solutions of BSVIEs in $\mathcal{H}^{2}[0, T]$. In [7] and [15], the authors considered the existence and uniqueness of adapted solutions of $(2.3)(\psi(\cdot)$ is replaced with $\xi)$ in $\mathcal{H}_{t}^{2}[0, T]$.

We give the following assumption on $g$ for BSVIE (1.2):

(H1) Let $g: \Delta^{c} \times \mathbb{R}^{m} \times \mathbb{R}^{m \times d} \times \mathbb{R}^{m \times d} \times \Omega \rightarrow \mathbb{R}^{m}$ be $\mathcal{B}\left(\Delta^{c} \times R^{m} \times \mathbb{R}^{m \times d} \times\right.$ $\left.\mathbb{R}^{m \times d}\right) \otimes \mathcal{F}_{T}$-measurable such that $s \rightarrow g(t, s, y, z, \zeta)$ is $\mathcal{F}_{s}$-progressively measurable for all $(t, y, z, \zeta) \in[0, T] \times \mathbb{R}^{m} \times \mathbb{R}^{m \times d} \times \mathbb{R}^{m \times d}$, furthermore, $g$ satisfies the Lipschitz conditions with stochastic coefficient, i.e., for any $y, \bar{y} \in \mathbb{R}^{m}, z$, $\bar{z}, \zeta, \bar{\zeta} \in \mathbb{R}^{m \times d}$

$$
\begin{aligned}
& |g(t, s, y, z, \zeta)-g(t, s, \bar{y}, \bar{z}, \bar{\zeta})| \\
\leq & L(t, s) \alpha(s)(|y-\bar{y}|+|z-\bar{z}|+|\zeta-\bar{\zeta}|), \quad(t, s) \in \Delta^{c},
\end{aligned}
$$

where $\alpha(\cdot)$ is an adapted process such that $\alpha(\cdot) \geq 1$, and $L(t, s)$ is a deterministic non-negative function with $(t, s) \in \Delta^{c}$. Given $p \in(1,2)$, suppose that $A(\cdot)$ is integrable process such that

$$
A(t)=\int_{0}^{t} \alpha^{\frac{2 p}{2-p}}(s) d s, \quad t \in[0, T] .
$$

Furthermore,

$$
E \int_{0}^{T} \int_{t}^{T} e^{\beta A(s)}\left|g_{0}(t, s)\right|^{2} d s d t<\infty \text {, where } g_{0}(t, s) \equiv g(t, s, 0,0,0) .
$$

\section{Main results for M-solutions and adapted solutions}

\subsection{A basic estimate for solutions of BSVIEs}

In this subsection, inspired by the method of estimating the adapted solutions of BSDEs in [5], we give an important lemma. 
Lemma 3.1. We consider the following simple BSVIE

$$
Y(t)=\psi(t)+\int_{t}^{T} f(t, s) d s-\int_{t}^{T} Z(t, s) d W(s), \quad t \in[0, T],
$$

where $\psi(\cdot) \in L_{\mathcal{F}_{T}}^{2, \beta}[0, T]$, and $f \in L^{2}\left(0, T ; L_{\mathbb{F}}^{2}\left(t, T ; \mathbb{R}^{m}\right)\right)$ such that

$$
E \int_{0}^{T} \int_{t}^{T} e^{\beta A(s)}|f(t, s)|^{2} d s d t<\infty
$$

Then (1.2) admits a unique adapted solution $(Y(\cdot), Z(\cdot, \cdot)) \in \mathcal{H}_{t}^{2, \beta}[0, T]$, and the following estimate holds, for $p \in[1,2)$,

$$
\begin{aligned}
& E \int_{0}^{T} e^{\beta A(s)}|Y(s)|^{2} d s+E \int_{0}^{T} \int_{t}^{T} e^{\beta A(s)}|Z(t, s)|^{2} d s d t \\
\leq & C E e^{\beta A(T)} \int_{0}^{T}|\psi(t)|^{2} d t+C E \int_{0}^{T} e^{\beta A(t)}\left|\int_{t}^{T} f(t, s) d s\right|^{2} d t \\
& +C E \int_{0}^{T} \int_{t}^{T} \beta \alpha^{\frac{2 p}{2-p}}(s) e^{\beta A(s)}\left|\int_{s}^{T} f(t, u) d u\right|^{2} d s d t .
\end{aligned}
$$

Furthermore,

$$
\begin{gathered}
E \int_{0}^{T} e^{\beta A(s)}|Y(s)|^{2} d s+E \int_{0}^{T} \int_{t}^{T} e^{\beta A(s)}|Z(t, s)|^{2} d s d t \\
\leq C E e^{\beta A(T)} \int_{0}^{T}|\psi(t)|^{2} d t+\frac{C}{\beta} E \int_{0}^{T} \int_{t}^{T} e^{\beta A(s)} \frac{|f(t, s)|^{2}}{\alpha^{\frac{2 p}{2-p}}(s)} d s d t .
\end{gathered}
$$

Hereafter $C$ is a generic positive constant which may be different from line to line.

Proof. Consider a family of BSDEs with parameters $t$ on $[0, T]$ in the following form:

$$
\lambda(t, r)=\psi(t)+\int_{r}^{T} f(t, s) d s-\int_{r}^{T} \mu(t, s) d W(s), \quad r \in[t, T] .
$$

By the classical existence and uniqueness theorem of BSDEs in [11], there exists a unique adapted solution $(\lambda(t, \cdot), \mu(t, \cdot))$ on $[t, T]$, for every $t \in[0, T]$. Let $Y(t)=\lambda(t, t)$ with $t \in[0, T], Z(t, s)=\mu(t, s)$ with $0 \leq t \leq s \leq T$. Then we obtain the existence of the adapted solution for (3.7). From (3.7), it follows that, $\forall r \in[t, T]$,

$$
\left\{\begin{array}{l}
\lambda(t, r)=E^{\mathcal{F}_{r}}\left(\psi(t)+\int_{r}^{T} f(t, s) d s\right) \\
\int_{r}^{T} Z(t, s) d W(s)=\int_{r}^{T} \mu(t, s) d W(s)=\psi(t)+\int_{r}^{T} f(t, s) d s-\lambda(t, r) .
\end{array}\right.
$$


Especially when $r=t$, we obtain that

$$
\int_{t}^{T} Z(t, s) d W(s)=\int_{t}^{T} \mu(t, s) d W(s)=\psi(t)+\int_{t}^{T} f(t, s) d s-Y(t) .
$$

Now we estimate

$$
E \int_{0}^{T} e^{\beta A(s)}|Y(s)|^{2} d s+E \int_{0}^{T} \int_{t}^{T} e^{\beta A(s)}|Z(t, s)|^{2} d s d t .
$$

By Cauchy-Schwarz inequality we deduce that

$$
\begin{aligned}
\left|\int_{s}^{T} f(t, u) d u\right|^{2} & =\left|\int_{s}^{T} e^{\frac{-\gamma A(u)}{2}} \alpha^{\frac{p}{2-p}}(u) e^{\frac{\gamma A(u)}{2}} \frac{f(t, u)}{\alpha^{\frac{p}{2-p}}(u)} d u\right|^{2} \\
& \leq \int_{s}^{T} e^{-\gamma A(u)} \alpha^{\frac{2 p}{2-p}}(u) d u \cdot \int_{s}^{T} e^{\gamma A(u)} \frac{|f(t, u)|^{2}}{\alpha^{\frac{2 p}{2-p}}(u)} d u \\
& \leq \frac{1}{\gamma} e^{-\gamma A(s)} \int_{s}^{T} e^{\gamma A(u)} \frac{|f(t, u)|^{2}}{\alpha^{\frac{2 p}{2-p}}(u)} d u, \quad 0 \leq s \leq t \leq T,
\end{aligned}
$$

where $\gamma=\frac{\beta}{2}$ or $\beta$. By taking $\gamma=\frac{\beta}{2}$ in (3.9), we see that

$$
\begin{aligned}
& \int_{t}^{T} \beta \alpha^{\frac{2 p}{2-p}}(s) e^{\beta A(s)}\left|\int_{s}^{T} f(t, u) d u\right|^{2} d s \\
\leq & \frac{4}{\beta} \int_{t}^{T} \beta \alpha^{\frac{2 p}{2-p}} e^{\frac{\beta}{2} A(s)}\left(\int_{s}^{T} e^{\frac{\beta}{2} A(u)} \frac{|f(t, u)|^{2}}{\alpha^{\frac{2 p}{2-p}}(u)} d u\right) d \frac{s}{2} \\
= & \left.\left(\frac{4}{\beta} e^{\frac{\beta}{2} A(s)} \cdot \int_{s}^{T} e^{\frac{\beta}{2} A(u)} \frac{|f(t, u)|^{2}}{\alpha^{\frac{2 p}{2-p}}(u)} d u\right)\right|_{t} ^{T}+\frac{4}{\beta} \int_{t}^{T} e^{\beta A(s)} \frac{|f(t, s)|^{2}}{\alpha^{\frac{2 p}{2-p}}(s)} d s \\
\leq & \frac{4}{\beta} \int_{t}^{T} e^{\beta A(s)} \frac{|f(t, s)|^{2}}{\alpha^{\frac{2 p}{2-p}}(s)} d s .
\end{aligned}
$$

Therefore,

$$
\begin{aligned}
& E \int_{0}^{T} \int_{t}^{T} \beta \alpha^{\frac{2 p}{2-p}} e^{\beta A(s)}\left|\int_{s}^{T} f(t, u) d u\right|^{2} d s d t \\
\leq & \frac{4}{\beta} E \int_{0}^{T} \int_{t}^{T} e^{\beta A(s)} \frac{|f(t, s)|^{2}}{\alpha^{\frac{2 p}{2-p}}(s)} d s d t .
\end{aligned}
$$

We also obtain the following result by taking $s=t$ and $\gamma=\beta$ in (3.9),

$$
E \int_{0}^{T} e^{\beta A(t)}\left|\int_{t}^{T} f(t, u) d u\right|^{2} d t \leq \frac{1}{\beta} E \int_{0}^{T} \int_{t}^{T} e^{\beta A(u)} \frac{|f(t, u)|^{2}}{\alpha^{\frac{2 p}{2-p}}(u)} d u d t .
$$


GENERAL BACKWARD STOCHASTIC VOLTERRA INTEGRAL EQUATIONS 1309

At first we estimate

$$
E \int_{0}^{T} \int_{t}^{T} e^{\beta A(s)}|Z(t, s)|^{2} d s d t .
$$

It is easy to see that

$$
\begin{aligned}
& \int_{r}^{T} \beta \alpha^{\frac{2 p}{2-p}}(s) e^{\beta A(s)}\left(\int_{s}^{T}|Z(t, u)|^{2} d u\right) d s \\
= & \left.\left(e^{\beta A(s)} \cdot \int_{s}^{T}|Z(t, u)|^{2} d u\right)\right|_{r} ^{T}+\int_{r}^{T} e^{\beta A(s)}|Z(t, s)|^{2} d s .
\end{aligned}
$$

For any $t \in[0, T]$, we can rewrite (3.11) after taking $r=t$,

$$
\begin{aligned}
& E \int_{0}^{T} \int_{t}^{T} e^{\beta A(s)}|Z(t, s)|^{2} d s d t \\
= & E \int_{0}^{T}\left[\int_{t}^{T} \beta \alpha^{\frac{2 p}{2-p}}(s) e^{\beta A(s)} \int_{s}^{T}|Z(t, u)|^{2} d u d s+e^{\beta A(t)} \int_{t}^{T}|Z(t, u)|^{2} d u\right] d t .
\end{aligned}
$$

Now we give an estimate to the second expression in the right part of (3.12)

$$
\begin{aligned}
& E \int_{0}^{T} e^{\beta A(t)} \int_{t}^{T}|Z(t, u)|^{2} d u d t \\
= & E \int_{0}^{T} E\left(e^{\beta A(t)} \int_{t}^{T}|Z(t, u)|^{2} d u \mid \mathcal{F}_{t}\right) d t \\
= & E \int_{0}^{T} e^{\beta A(t)} E\left(\left(\int_{t}^{T} Z(t, u) d W(u)\right)^{2} \mid \mathcal{F}_{t}\right) d t \\
= & E \int_{0}^{T} e^{\beta A(t)} E\left(\left(\psi(t)+\int_{t}^{T} f(t, u) d u-Y(t)\right)^{2} \mid \mathcal{F}_{t}\right) d t \\
\leq & 3 E \int_{0}^{T} e^{\beta A(t)}|\psi(t)|^{2} d t+3 E \int_{0}^{T} e^{\beta A(t)}\left|\int_{t}^{T} f(t, u) d u\right|^{2} d t \\
& +3 E \int_{0}^{T} e^{\beta A(t)}|Y(t)|^{2} d t \\
\leq & 3 E \int_{0}^{T} e^{\beta A(t)}|\psi(t)|^{2} d t+\frac{3}{\beta} E \int_{0}^{T} \int_{t}^{T} e^{\beta A(u)} \frac{|f(t, u)|^{2}}{\alpha^{\frac{2 p}{2-p}}(u)} d u d t \\
& +3 E \int_{0}^{T} e^{\beta A(t)}|Y(t)|^{2} d t .
\end{aligned}
$$


Obviously, we can use the similar method as (3.13) to estimate the first expression in the right part of (3.12) as follows:

$$
\begin{aligned}
& E \int_{0}^{T} \int_{t}^{T} \beta \alpha^{\frac{2 p}{2-p}}(s) e^{\beta A(s)}\left(\int_{s}^{T}|Z(t, u)|^{2} d u\right) d s d t \\
\leq & 3 E \int_{0}^{T} \int_{t}^{T} \beta \alpha^{\frac{2 p}{2-p}}(s) e^{\beta A(s)}|\psi(t)|^{2} d s d t \\
& +3 E \int_{0}^{T} \int_{t}^{T} \beta \alpha^{\frac{2 p}{2-p}}(s) e^{\beta A(s)}\left|\int_{s}^{T} f(t, u) d u\right|^{2} d s d t \\
& +3 E \int_{0}^{T} \int_{t}^{T} \beta \alpha^{\frac{2 p}{2-p}}(s) e^{\beta A(s)}|\lambda(t, s)|^{2} d s d t .
\end{aligned}
$$

For the second expression in the right part of (3.14), (3.10) implies that

$$
\begin{aligned}
& 3 E \int_{0}^{T} \int_{t}^{T} \beta \alpha^{\frac{2 p}{2-p}}(s) e^{\beta A(s)}\left|\int_{s}^{T} f(t, u) d u\right|^{2} d s d t \\
\leq & \frac{12}{\beta} E \int_{0}^{T} \int_{t}^{T} e^{\beta A(s)} \frac{|f(t, s)|^{2}}{\alpha^{\frac{2 p}{2-p}}(s)} d s d t .
\end{aligned}
$$

Now let us to estimate the third expression of (3.14). Noting that

$$
\lambda(t, s)=E^{\mathcal{F}_{s}}\left(\psi(t)+\int_{s}^{T} f(t, u) d u\right), \quad t \leq s,
$$

we deduce that,

$$
|\lambda(t, s)|^{2} \leq 2 E^{\mathcal{F}_{s}}|\psi(t)|^{2}+2 E^{\mathcal{F}_{s}}\left|\int_{s}^{T} f(t, u) d u\right|^{2},
$$

and

$$
\begin{gathered}
\\
3 E \int_{0}^{T} \int_{t}^{T} \beta \alpha^{\frac{2 p}{2-p}}(s) e^{\beta A(s)}|\lambda(t, s)|^{2} d s d t \\
\leq 6 E \int_{0}^{T}\left[e^{\beta A(T)}|\psi(t)|^{2}+\int_{t}^{T} \beta \alpha^{\frac{2 p}{2-p}}(s) e^{\beta A(s)}\left|\int_{s}^{T} f(t, u) d u\right|^{2} d s\right] d t \\
(3.16) \leq 6 E e^{\beta A(T)} \int_{0}^{T}|\psi(t)|^{2} d t+\frac{24}{\beta} E \int_{0}^{T} \int_{t}^{T} e^{\beta A(s)} \frac{|f(t, s)|^{2}}{\alpha^{\frac{2 p}{2-p}}(s)} d s d t .
\end{gathered}
$$

(3.14), (3.15), (3.16) imply that

$$
E \int_{0}^{T} \int_{t}^{T} \beta \alpha^{\frac{2 p}{2-p}}(s) e^{\beta A(s)}\left(\int_{s}^{T}|Z(t, u)|^{2} d u\right) d s d t
$$




$$
\leq \frac{36}{\beta} E \int_{0}^{T} \int_{t}^{T} e^{\beta A(s)} \frac{|f(t, s)|^{2}}{\alpha^{\frac{2 p}{2-p}}(s)} d s d t+9 E e^{\beta A(T)} \int_{0}^{T}|\psi(t)|^{2} d t .
$$

By (3.12), (3.13), (3.17) we also see that

$$
\begin{aligned}
& E \int_{0}^{T} \int_{t}^{T} e^{\beta A(s)}|Z(t, s)|^{2} d s d t \\
\leq & 12 E e^{\beta A(T)} \int_{0}^{T}|\psi(t)|^{2} d t+3 E \int_{0}^{T} e^{\beta A(t)}|Y(t)|^{2} d t \\
& +\frac{39}{\beta} E \int_{0}^{T} \int_{t}^{T} e^{\beta A(s)} \frac{|f(t, s)|^{2}}{\alpha^{\frac{2 p}{2-p}}(s)} d s d t .
\end{aligned}
$$

Due to $Y(t)=E^{\mathcal{F}_{t}}\left(\psi(t)+\int_{t}^{T} f(t, s) d s\right)$, we have

$$
\begin{aligned}
& E \int_{0}^{T} e^{\beta A(t)}|Y(t)|^{2} d t \\
\leq & 2 E \int_{0}^{T} e^{\beta A(t)}|\psi(t)|^{2} d t+2 E \int_{0}^{T} e^{\beta A(t)}\left|\int_{t}^{T} f(t, s) d s\right|^{2} d t \\
\leq & 2 E \int_{0}^{T} e^{\beta A(t)}|\psi(t)|^{2} d t+\frac{2}{\beta} E \int_{0}^{T} \int_{t}^{T} e^{\beta A(s)} \frac{|f(t, s)|^{2}}{\alpha^{\frac{2 p}{2-p}}(s)} d s d t .
\end{aligned}
$$

Eventually we obtain that

$$
\begin{gathered}
E \int_{0}^{T} e^{\beta A(s)}|Y(s)|^{2} d s+E \int_{0}^{T} \int_{t}^{T} e^{\beta A(s)}|Z(t, s)|^{2} d s d t \\
\leq 20 E e^{\beta A(T)} \int_{0}^{T}|\psi(t)|^{2} d t+11 E \int_{0}^{T} e^{\beta A(t)}\left|\int_{t}^{T} f(t, s) d s\right|^{2} d t \\
\quad+9 E \int_{0}^{T} \int_{t}^{T} \beta \alpha^{\frac{2 p}{2-p}}(s) e^{\beta A(s)}\left|\int_{s}^{T} f(t, u) d u\right|^{2} d s d t .
\end{gathered}
$$

Furthermore, it follows that

$$
\begin{aligned}
& E \int_{0}^{T} e^{\beta A(t)}|Y(t)|^{2} d t+E \int_{0}^{T} \int_{t}^{T} e^{\beta A(s)}|Z(t, s)|^{2} d s d t \\
\leq & 20 E e^{\beta A(T)} \int_{0}^{T}|\psi(t)|^{2} d t+\frac{47}{\beta} E \int_{0}^{T} \int_{t}^{T} e^{\beta A(s)} \frac{|f(t, s)|^{2}}{\alpha^{\frac{2 p}{2-p}}(s)} d s d t .
\end{aligned}
$$

It is easy to see that (3.5) or (3.6) implies the uniqueness of solutions for (3.4).

Remark 1. If we define $Z(t, s)(0 \leq s<t \leq T)$ by the following relation

$$
Y(t)=E Y(t)+\int_{0}^{t} Z(t, s) d W(s), \quad \forall t \in[0, T]
$$


Then BSVIE (3.4) admits a unique M-solution in $\mathcal{H}^{2, \beta}[0, T]$.

Remark 2. Under the same conditions as Lemma 3.1, we can also get a slight sharper estimate for $(Y, Z)$ than $(3.6)$ as follows,

$$
\begin{gathered}
E e^{\beta A(t)}|Y(t)|^{2}+E \int_{t}^{T} e^{\beta A(s)}|Z(t, s)|^{2} d s \\
\leq E e^{\beta A(T)}|\psi(t)|^{2}+\frac{C}{\beta} E \int_{t}^{T} e^{\beta A(s)} \frac{|f(t, s)|^{2}}{\alpha^{\frac{2 p}{2-p}}(s)} d s,
\end{gathered}
$$

with $p \in[1,2)$.

\subsection{The Lipschitz case}

In this subsection, comparing with the method in [19], we give the existence and uniqueness of adapted M-solutions under Lipschitz conditions by a much more convenient method. Before it, we need an inequality which will play an important role in the following proof. Given $1 \leq p<2, f$ is a deterministic function, suppose that

$$
\int_{r}^{T} e^{\tau A(s) \frac{2}{p}} \frac{|f(t, s)|^{2}}{\alpha^{2}(s)} d s<\infty, \quad t, r \in[0, T], \tau>0
$$

then we have

$$
\begin{aligned}
& \left(\int_{r}^{T}|f(t, s)|^{p} d s\right)^{\frac{2}{p}} \\
= & \left(\int_{r}^{T} e^{-\tau A(s)} \alpha^{p}(s) e^{\tau A(s)} \frac{|f(t, s)|^{p}}{\alpha^{p}(s)} d s\right)^{\frac{2}{p}} \\
\leq & \left(\int_{r}^{T} e^{-\tau A(s) \frac{2}{2-p}} \alpha^{\frac{2 p}{2-p}}(s) d s\right)^{\frac{2-p}{p}}\left(\int_{r}^{T} e^{\tau A(s) \frac{2}{p}} \frac{|f(t, s)|^{2}}{\alpha^{2}(s)} d s\right) \\
\leq & \left(\frac{2-p}{2 \tau}\right)^{\frac{2-p}{p}} e^{-\tau A(r) \frac{2}{p}} \int_{r}^{T} e^{\tau A(s) \frac{2}{p}} \frac{|f(t, s)|^{2}}{\alpha^{2}(s)} d s .
\end{aligned}
$$

Particularly, let $\tau=\frac{p}{2} \beta$, we arrive at

$$
\left(\int_{r}^{T}|f(t, s)|^{p} d s\right)^{\frac{2}{p}} \leq\left(\frac{2-p}{p}\right)^{\frac{2-p}{p}}\left(\frac{1}{\beta}\right)^{\frac{2-p}{p}} e^{-\beta A(r)} \int_{r}^{T} e^{\beta A(s)} \frac{|f(t, s)|^{2}}{\alpha^{2}(s)} d s .
$$

Theorem 3.2. Let $(\mathrm{H} 1)$ hold, $\psi(\cdot) \in L_{\mathcal{F}_{T}}^{2, \beta}[0, T]$, and $L(\cdot, \cdot)$ satisfy

$$
\sup _{t \in[0, T]}\left(\int_{t}^{T} L^{q}(t, s) d s\right)^{\frac{2}{q}}<\infty, \quad \frac{1}{p}+\frac{1}{q}=1
$$

$\alpha(\cdot)$ is deterministic, then (1.2) admits a unique adapted $M$-solution in $\mathcal{H}^{2}[0, T]$. 
Proof. Since $A(\cdot)$ is deterministic, by the definition of adapted M-solutions, it is easy to see that

$$
E \int_{0}^{T} \int_{0}^{t} e^{\beta A(s)}|Z(t, s)|^{2} d s d t \leq E \int_{0}^{T} e^{\beta A(t)}|Y(t)|^{2} d t .
$$

As a consequence,

$$
\begin{aligned}
& E \int_{0}^{T} e^{\beta A(t)}|Y(t)|^{2} d t+E \int_{0}^{T} \int_{0}^{T} e^{\beta A(s)}|Z(t, s)|^{2} d s d t \\
\leq & 2 E \int_{0}^{T} e^{\beta A(t)}|Y(t)|^{2} d t+E \int_{0}^{T} \int_{t}^{T} e^{\beta A(s)}|Z(t, s)|^{2} d s d t .
\end{aligned}
$$

Let $\mathcal{M}^{2, \beta}[0, T]$ be the space of all $(y(\cdot), z(\cdot, \cdot)) \in \mathcal{H}^{2, \beta}[0, T]$ such that

$$
y(t)=E y(t)+\int_{0}^{t} z(t, s) d W(s), \quad t \in[0, T] .
$$

Clearly, it is a nonempty closed subspace of $\mathcal{H}^{2, \beta}[0, T]$. Now we consider the following BSVIE:

$$
Y(t)=\psi(t)+\int_{t}^{T} g(t, s, y(s), z(t, s), z(s, t)) d s-\int_{t}^{T} Z(t, s) d W(s), \quad t \in[0, T],
$$

for any $\psi(\cdot) \in L_{T}^{2, \beta}[0, T]$ and $(y(\cdot), z(\cdot, \cdot)) \in \mathcal{M}^{2, \beta}[0, T]$. Hence by Remark 1 we know that $(3.21)$ admits a unique M-solution $(Y(\cdot), Z(\cdot, \cdot)) \in \mathcal{M}^{2, \beta}[0, T]$, and we can define a mapping $\Theta: \mathcal{M}^{2, \beta}[0, T] \rightarrow \mathcal{M}^{2, \beta}[0, T]$ by

$$
\Theta(y(\cdot), z(\cdot, \cdot))=(Y(\cdot), Z(\cdot, \cdot)), \quad \forall(y(\cdot), z(\cdot, \cdot)) \in \mathcal{M}^{2, \beta}[0, T] .
$$

Let $(\bar{y}(\cdot), \bar{z}(\cdot, \cdot)) \in \mathcal{M}^{2, \beta}[0, T]$ and $\Theta(\bar{y}(\cdot), \bar{z}(\cdot, \cdot))=(\bar{Y}(\cdot), \bar{Z}(\cdot, \cdot))$. It follows from (3.5) in Lemma 3.1 and (3.20) above that,

$$
\begin{aligned}
& E \int_{0}^{T} e^{\beta A(t)}|Y(t)-\bar{Y}(t)|^{2} d t+E \int_{0}^{T} \int_{0}^{T} e^{\beta A(s)}|Z(t, s)-\bar{Z}(t, s)|^{2} d s d t \\
\leq & C E \int_{0}^{T}\left[e^{\beta A(t)}\left|\int_{t}^{T} H(t, s) d s\right|^{2}+\int_{t}^{T} \beta \alpha^{\frac{2 p}{2-p}}(s) e^{\beta A(s)}\left|\int_{s}^{T} H(t, u) d u\right|^{2} d s\right] d t \\
\leq & C E \int_{0}^{T}\left[e^{\beta A(t)}\left|\int_{t}^{T} G(t, s) d s\right|^{2}+\int_{t}^{T} \beta \alpha^{\frac{2 p}{2-p}}(s) e^{\beta A(s)}\left|\int_{s}^{T} G(t, u) d u\right|^{2} d s\right] d t \\
\leq & C E \int_{0}^{T} e^{\beta A(t)}\left(\int_{t}^{T} L^{q}(t, s) d s\right)^{\frac{2}{q}}\left(\int_{t}^{T} U^{p}(t, s) d s\right)^{\frac{2}{p}} d t \\
& +C E \int_{0}^{T} \int_{t}^{T} \beta \alpha^{\frac{2 p}{2-p}}(s) e^{\beta A(s)}\left(\int_{s}^{T} L^{q}(t, u) d u\right)^{\frac{2}{q}}\left(\int_{s}^{T} U^{p}(t, u) d u\right)^{\frac{2}{p}} d s d t,
\end{aligned}
$$


where

$$
\begin{aligned}
H(t, s) & =g(t, s, y(s), z(t, s), z(s, t))-g(t, s, \bar{y}(s), \bar{z}(t, s), \bar{z}(s, t)), \\
G(t, s) & =L(t, s) \alpha(s)(|y(s)-\bar{y}(s)|+|z(t, s)-\bar{z}(t, s)|+|z(s, t)-\bar{z}(s, t)|), \\
U(t, s) & =\alpha(s)(|y(s)-\bar{y}(s)|+|z(t, s)-\bar{z}(t, s)|+|z(s, t)-\bar{z}(s, t)|) .
\end{aligned}
$$

From inequality (3.19) above, we have

$$
\begin{aligned}
& E \int_{0}^{T}\left[e^{\beta A(t)}\left(\int_{t}^{T} U^{p}(t, s) d s\right)^{\frac{2}{p}}\right. \\
& \left.+\int_{t}^{T} \beta \alpha^{\frac{2 p}{2-p}}(s) e^{\beta A(s)}\left(\int_{s}^{T} U^{p}(t, u) d u\right)^{\frac{2}{p}} d s\right] d t \\
& \leq C\left(\frac{1}{\beta}\right)^{\frac{2-p}{p}} E \int_{0}^{T} \int_{t}^{T} e^{\beta A(s)}|y(s)-\bar{y}(s)|^{2} d s d t \\
& +C\left(\frac{1}{\beta}\right)^{\frac{2-p}{p}} E \int_{0}^{T} \int_{t}^{T} e^{\beta A(s)}|z(t, s)-\bar{z}(t, s)|^{2} d s d t \\
& \quad+C\left(\frac{1}{\beta}\right)^{\frac{2-p}{p}} E \int_{0}^{T} \int_{t}^{T} e^{\beta A(s)}|z(s, t)-\bar{z}(s, t)|^{2} d s d t .
\end{aligned}
$$

Eventually we get

$$
\begin{aligned}
& E \int_{0}^{T} e^{\beta A(t)}|Y(t)-\bar{Y}(t)|^{2} d t+E \int_{0}^{T} \int_{0}^{T} e^{\beta A(s)}|Z(t, s)-\bar{Z}(t, s)|^{2} d s d t \\
\leq & C\left(\frac{1}{\beta}\right)^{\frac{2-p}{p}} E \int_{0}^{T} e^{\beta A(s)}|y(s)-\bar{y}(s)|^{2} d s \\
& +C\left(\frac{1}{\beta}\right)^{\frac{2-p}{p}} E \int_{0}^{T} \int_{t}^{T} e^{\beta A(s)}|z(t, s)-\bar{z}(t, s)|^{2} d s d t .
\end{aligned}
$$

Choosing a sufficient large number $\beta$ so that $C\left(\frac{1}{\beta}\right)^{\frac{2-p}{p}}<1$. Then the mapping $\Theta$ is contractive from $\mathcal{H}^{2, \beta}[0, T]$ onto itself. Since $A(s)$ is integrable and deterministic, it follows that BSVIE (1.2) admits a unique M-solution in $\mathcal{H}^{2}[0, T]$.

Remark 3. By (3.20), one can understand the reason for assuming $\alpha(\cdot)$ to be deterministic. In this case, $A$ is bounded, hence the norm of $\mathcal{H}^{2, \beta}[0, T]$ and $L_{\mathcal{F}_{T}}^{2, \beta}$ are equivalent to the norm of $\mathcal{H}^{2}[0, T]$ and $L_{\mathcal{F}_{T}}^{2}$ respectively, thus BSVIE (1.2) admits a unique M-solution in $\mathcal{H}^{2}[0, T]$. As a result, we can finish the proof of existence and uniqueness of M-solutions in $\mathcal{H}^{2}[0, T]$ with only one step, which is much more convenient than the four steps in [19]. 
Remark 4. In our setting we only impose some integrable conditions on $L$, i.e.,

$$
\sup _{t \in[0, T]} \int_{t}^{T} L^{q}(t, s) d s<\infty, \quad q>2 .
$$

It is equivalent to the requirement in [19] where $q$ above is replaced by $2+\epsilon$ with $\epsilon$ be a positive constant. Note that (3.19) plays a key role in this procedure.

Similarly we can obtain the existence and uniqueness of the adapted solutions for $(2.3)$ in $\mathcal{H}_{t}^{2}[0, T]$. In order to estimate the value of $Z(t, s)$ with $(t, s) \in \Delta[0, T]$, we suppose $A(\cdot)$ to be deterministic. However, as to adapted solution in in $\mathcal{H}_{t}^{2}[0, T]$, such requirement is not needed.

Theorem 3.3. Let $(\mathrm{H} 1)$ hold, $\psi(\cdot) \in L_{\mathcal{F}_{T}}^{2}[0, T]$, and $\sup _{t \in[0, T]}\left(\int_{t}^{T} L^{q}(t, s) d s\right)^{\frac{2}{q}}$ $<\infty$. Moreover $A(\cdot)$ is bounded. Then BSVIE (2.3) admits a unique adapted solution in $\mathcal{H}_{t}^{2}[0, T]$.

Proof. We can get the result by similar ideas as in Theorem 3.2 above, so we omit it.

\subsection{The non-Lipschitz case}

In this subsection, we will consider the unique existence of adapted Msolution of $(1.2)$ in $\mathcal{H}^{2}[0, T]$ and adapted solution of $(2.3)$ in $\mathcal{H}_{t}^{2}[0, T]$ under non-Lipschitz conditions. We assume that

(H2) For all $y, \bar{y} \in \mathbb{R}^{m}, z, \bar{z}, \zeta, \bar{\zeta} \in \mathbb{R}^{m \times d}$, and $(t, s) \in \Delta^{c}$

$$
\begin{aligned}
& |g(t, s, y, z, \zeta)-g(t, s, \bar{y}, \bar{z}, \bar{\zeta})| \\
\leq & L(t, s) \alpha(s)\left(\left(\rho\left(|y-\bar{y}|^{2}\right)\right)^{\frac{1}{2}}+|z-\bar{z}|+|\zeta-\bar{\zeta}|\right),
\end{aligned}
$$

where $\rho$ is an increasing concave function from $\mathbb{R}_{+}$to $\mathbb{R}_{+}$such that $\rho(0)=0$, and $\int_{0_{+}} \frac{d u}{\rho(u)}=\infty . L(t, s)$ is a deterministic non-negative function.

Since $\rho$ is concave and $\rho(0)=0$, one can find a pair of positive constants $a$ and $b$ such that $\rho(u) \leq a+b u$, for all $u \geq 0$. Ren [13] studied well-posedness of adapted M-solution of (1.2) under non-Lipschitz condition in infinite dimensional space. However, there are some problems that should be pointed out. Let us consider the following example.

Example 1. Let $L(t, s)=\sqrt{\frac{2}{T-t}}$ with $0 \leq t<T, t \leq s$, and $L(t, s)=0$ with $t=0, t \leq s$, thus we have

$$
\sup _{t \in[0, T]} \int_{t}^{T} L^{2}(t, s) d s=2<\infty,
$$

which satisfies the assumption (H3) on p. 4 in [13]. However, there does not exist a sequence $0=T_{0}<T_{2}<\cdots<T_{k-1}<T_{k}=T$ such that

$$
\sup _{t \in\left[T_{i-1}, T_{i}\right]} \int_{t}^{T_{i}} L^{2}(t, s) d s \leq 1-\delta
$$


with $\delta \in(0,1)$. In fact, in such case there is no $T_{k-1}$ satisfying the above condition.

By Example 1, it is clear that there exists functions satisfying (H3) in [13] which does not have a split procedure as above. In this paper we will specify some other assumptions on the coefficients which is more weaker than ones in [13]. On the other hand, for the unique solvability of M-solutions for (1.2) under non-Lipschitz conditions, one will meet an expression $E \int_{t}^{T}|Z(s, t)|^{2} d s$ which is hard to estimate. Therefore we cannot use the method in [15] (see p. 10 in [13]) directly to treat it. Some other new tricks should be proposed to overcome the difficulty in such general setting. Firstly, we need to introduce a lemma, i.e., a new type of Jensen's inequality, which is indispensable in the following theorem.

Lemma 3.4. Let $f(t):[0, T] \rightarrow \mathbb{R}^{+}$satisfy $\int_{0}^{T} f(t) d t<\infty$ and $c(x): \mathbb{R} \rightarrow \mathbb{R}$ be a concave function. Then we have

$$
\frac{1}{T-t} \int_{t}^{T} c(f(s)) d s \leq c\left(\frac{1}{T-t} \int_{t}^{T} f(s) d s\right), \quad \forall t \in[0, T] .
$$

Proof. Obviously $-c(x)$ is a convex function, for fixed $x \in \mathbb{R}, \forall y_{1}>x, y_{2}<x$, we have (see p. 544 in [12]).

$$
\frac{-c\left(y_{1}\right)+c(x)}{y_{1}-x} \geq-c_{+}^{\prime}(x) \geq-c_{-}^{\prime}(x) \geq \frac{-c\left(y_{2}\right)+c(x)}{y_{2}-x},
$$

thus there exists a $k \in\left[-c_{-}^{\prime}(x),-c_{+}^{\prime}(x)\right]$ so that $\forall y \in \mathbb{R},-c(y) \geq-c(x)+$ $k \cdot(y-x)$, i.e., $c(y) \leq c(x)-k(y-x)$. For any fixed $t \in[0, T], s \in[t, T]$, let $x=\frac{1}{T-t} \int_{t}^{T} f(s) d s, y=f(s)$, then

$$
c(f(s)) \leq c\left(\frac{1}{T-t} \int_{t}^{T} f(s) d s\right)-k \cdot\left(f(s)-\frac{1}{T-t} \int_{t}^{T} f(s) d s\right),
$$

thus the desired conclusion is obtained.

We are now ready to establish the main result of this subsection.

Theorem 3.5. Let $(\mathrm{H} 2)$ hold, $\psi(\cdot) \in L_{\mathcal{F}_{T}}^{2}[0, T], \alpha(\cdot)$ is deterministic, $L(t, s)$ satisfy $\sup _{t \in[0, T]}\left(\int_{t}^{T} L^{q}(t, s) d s\right)^{\frac{2}{q}}<\infty$. Then (1.2) admits a unique adapted $M$-solution in $\mathcal{H}^{2}[0, T]$.

Proof. Uniqueness: Let $\left(Y_{i}, Z_{i}\right) \in \mathcal{H}^{2}[0, T](i=1,2)$ be any two adapted Msolutions. For $t, s \in[0, T]$, by defining

$$
\widehat{Y}(t)=Y_{1}(t)-Y_{2}(t), \quad \widehat{Z}(t, s)=Z_{1}(t, s)-Z_{2}(t, s),
$$

it follows that

$$
\widehat{Y}(t)+\int_{t}^{T} \widehat{Z}(t, s) d W(s)
$$


GENERAL BACKWARD STOCHASTIC VOLTERRA INTEGRAL EQUATIONS 1317

$=\int_{t}^{T}\left[g\left(t, s, Y_{1}(s), Z_{1}(t, s), Z_{1}(s, t)\right)-g\left(t, s, Y_{2}(s), Z_{2}(t, s), Z_{2}(s, t)\right)\right] d s$.

Note that $\widehat{Y}(T)=0$. For arbitrary $u \in[0, T)$, we can obtain the following results in the same way as in Theorem 3.2 ,

$$
\begin{aligned}
& E \int_{u}^{T} e^{\beta A(t)}|\widehat{Y}(t)|^{2} d t+E \int_{u}^{T} \int_{t}^{T} e^{\beta A(s)}|\widehat{Z}(t, s)|^{2} d s d t \\
\leq & C\left(\frac{1}{\beta}\right)^{\frac{2-p}{p}} E \int_{u}^{T} \int_{t}^{T} e^{\beta A(s)} \rho\left(|\widehat{Y}(s)|^{2}\right) d s d t \\
& +C\left(\frac{1}{\beta}\right)^{\frac{2-p}{p}}\left[E \int_{u}^{T} \int_{t}^{T} e^{\beta A(s)}|\widehat{Z}(t, s)|^{2} d s d t+E \int_{u}^{T} e^{\beta A(t)}|\widehat{Y}(t)|^{2} d t\right] .
\end{aligned}
$$

By choosing a suitable $\beta$, it holds that

$$
E \int_{u}^{T} e^{\beta A(t)}|\widehat{Y}(t)|^{2} d t \leq C E \int_{u}^{T} \int_{t}^{T} e^{\beta A(s)} \rho\left(|\widehat{Y}(s)|^{2}\right) d s d t,
$$

consequently,

$$
\begin{aligned}
\frac{1}{T-u} E \int_{u}^{T}|\widehat{Y}(t)|^{2} d t & \leq C E \int_{u}^{T} \frac{1}{T-t} \int_{t}^{T} \rho\left(|\widehat{Y}(s)|^{2}\right) d s d t \\
& \leq C \int_{u}^{T} \rho\left(\frac{1}{T-t} \int_{t}^{T} E|\widehat{Y}(s)|^{2} d s\right) d t .
\end{aligned}
$$

Due to Bihari's inequality (see [3]) we get that

$$
\frac{1}{T-u} E \int_{u}^{T}|\widehat{Y}(t)|^{2} d t=0 \quad \forall u \in[0, T),
$$

thus $\widehat{Y}(t)=0$ as well as $\widehat{Z}(t, s)=0$, a.s., $\forall t, s \in[0, T]$.

Existence: Let $Y_{0}(t) \equiv 0$, and define recursively $\left(Y_{n}, Z_{n}\right)$ by the following equations with the help of Theorem 3.2

$$
Y_{n}(t)=\psi(t)+\int_{t}^{T} g\left(t, s, Y_{n-1}(s), Z_{n}(t, s), Z_{n}(s, t)\right) d s-\int_{t}^{T} Z_{n}(t, s) d W(s) .
$$

By setting

$$
\widehat{Y}_{n, k}(t)=Y_{n}(t)-Y_{k}(t) ; \quad \widehat{Z}_{n, k}(t, s)=Z_{n}(t, s)-Z_{k}(t, s), \quad t, s \in[0, T],
$$

and choosing a suitable $\beta$, we claim that

$$
\begin{aligned}
& E \int_{u}^{T} e^{\beta A(t)}\left|\widehat{Y}_{n, k}(t)\right|^{2} d t+E \int_{u}^{T} \int_{t}^{T} e^{\beta A(s)}\left|\widehat{Z}_{n, k}(t, s)\right|^{2} d s d t \\
\leq & C E \int_{u}^{T} \int_{t}^{T} e^{\beta A(s)} \rho\left(\left|\widehat{Y}_{n-1, k-1}(s)\right|^{2}\right) d s d t,
\end{aligned}
$$


then

$$
\frac{1}{T-u} E \int_{u}^{T}\left|\widehat{Y}_{n, k}(t)\right|^{2} d t \leq C \int_{u}^{T} \rho\left(\frac{1}{T-t} \int_{t}^{T} E\left|\widehat{Y}_{n-1, k-1}(s)\right|^{2} d s\right) d t .
$$

Set

$$
Q(u)=\limsup _{n, k \rightarrow \infty} E \int_{u}^{T}\left|\widehat{Y}_{n, k}(t)\right|^{2} d t, \quad u \in[0, T]
$$

then

$$
S(u)=\sup _{n \geq 0} E \int_{u}^{T}\left|Y_{n}(t)\right|^{2} d t, \quad u \in[0, T]
$$

is bounded. In fact, by the similar trick as in Theorem 3.2, it follows that

$$
\begin{aligned}
& E \int_{u}^{T} e^{\beta A(t)}\left|Y_{n}(t)\right|^{2} d t+E \int_{u}^{T} \int_{t}^{T} e^{\beta A(s)}\left|Z_{n}(t, s)\right|^{2} d s d t \\
\leq & C E \int_{u}^{T} e^{\beta A(t)}|\psi(t)|^{2} d t+C\left(\frac{1}{\beta}\right)^{\frac{2-p}{p}} E \int_{u}^{T} \int_{t}^{T} e^{\beta A(s)}\left|g_{0}(t, s)\right|^{2} d s d t \\
& +C\left(\frac{1}{\beta}\right)^{\frac{2-p}{p}} E \int_{u}^{T} \int_{t}^{T} e^{\beta A(s)}\left(a+b\left|Y_{n-1}(s)\right|^{2}\right) d s d t \\
& +C\left(\frac{1}{\beta}\right)^{\frac{2-p}{p}}\left[E \int_{u}^{T} \int_{t}^{T} e^{\beta A(s)}\left|Z_{n}(t, s)\right|^{2} d s d t+E \int_{u}^{T} e^{\beta A(t)}\left|Y_{n}(t)\right|^{2} d t\right] .
\end{aligned}
$$

Thus by choosing $\beta$, we have

$$
\begin{aligned}
E \int_{u}^{T}\left|Y_{n}(t)\right|^{2} d t \leq & C+C E \int_{u}^{T}|\psi(t)|^{2} d t \\
& +C E \int_{u}^{T} \int_{t}^{T}\left|g_{0}(t, s)\right|^{2} d s d t+C E \int_{u}^{T} \int_{t}^{T}\left|Y_{n-1}(s)\right|^{2} d s d t .
\end{aligned}
$$

In view of Gronwall's inequality we know that $S(u)$ is bounded. Then by Fatou's lemma, Bihari's inequality and noting that $\rho$ is increasing, we deduce that for almost every $u \in[0, T], Q(u)=0$, and it follows that

$$
\lim _{n, k \rightarrow \infty} E \int_{0}^{T}\left|Y_{n}(t)-Y_{k}(t)\right|^{2} d t=0
$$

hence there is a $Y$ such that

$$
\lim _{n \rightarrow \infty} E \int_{0}^{T}\left|Y_{n}(t)-Y(t)\right|^{2} d t=0 .
$$

Similarly there is a $Z$ such that

$$
\begin{aligned}
& \lim _{n \rightarrow \infty} E \int_{0}^{T} \int_{t}^{T}\left|Z_{n}(t, s)-Z(t, s)\right|^{2} d s d t=0, \\
& \lim _{n \rightarrow \infty} E \int_{0}^{T} \int_{0}^{t}\left|Z_{n}(t, s)-Z(t, s)\right|^{2} d s d t \leq \lim _{n \rightarrow \infty} E \int_{0}^{T}\left|Y_{n}(t)-Y(t)\right|^{2} d t=0 .
\end{aligned}
$$


By taking the limits for $(3.22)$, one can find that $(Y, Z)$ is an M-solution of (1.2).

As to the adapted solution of $(2.3)$ in $\mathcal{H}_{t}^{2}[0, T], \alpha(\cdot)$ can be allowed to be random, and we have:

Theorem 3.6. Let (H2) hold, $\psi(\cdot) \in L_{\mathcal{F}_{T}}^{2}[0, T], \sup _{t \in[0, T]}\left(\int_{t}^{T} L^{q}(t, s) d s\right)^{\frac{2}{q}}<$ $\infty, A^{*}(t)$ be bounded. Then BSVIE (2.3) admits a unique adapted solution in $\mathcal{H}_{t}^{2}[0, T]$.

Proof. The proof can be easily completed by using the similar ideas in Theorem 3.5 above, so we omit it.

When $\alpha(\cdot)$ is a constant and $\psi(\cdot)=\xi, g$ is independent of $Z(s, t)$, then we get the result in [15]:

Corollary 1. Let $(\mathrm{H} 2)$ hold, $r_{i}(s)=1, L(t, s)=k, k$ is a constant. Then (2.3) admits a unique adapted solution in $\mathcal{H}_{t}^{2}[0, T]$.

Remark 5. By means of Itô formula, the authors in [15] gave a critical estimate for

$$
e^{\beta t}|Y(t)|^{2}+E^{\mathcal{F}_{t}} \int_{t}^{T} e^{\beta s}|Z(t, s)|^{2} d s
$$

It is similar to the estimate in Remark 2 above which can be derived without the involving of Itô formula, and both of them are stronger than the estimate of the form

$$
E \int_{0}^{T} e^{\beta A(t)}|Y(t)|^{2} d t+E \int_{0}^{T} \int_{t}^{T} e^{\beta A(s)}|Z(t, s)|^{2} d s d t
$$

used in Theorems 3.2, 3.3, 3.5 and 3.6 above. However, both of the previous two estimates have certain drawbacks. In fact, if we use any one of the two estimates above to prove the existence and uniqueness of M-solution of (1.2) in $\mathcal{H}^{2}[0, T]$ and adapted solution of $(2.3)$ in $\mathcal{H}_{t}^{2}[0, T]$, it is easy to check the stronger conditions of $\sup _{t \in[0, T]} E|\psi(t)|^{2}<\infty$ and $\sup _{t \in[0, T]} E \int_{t}^{T}\left|g_{0}(t, s)\right|^{2} d s<\infty$ are required.

\section{Examples}

At last we want to give a simple example to show the unique existence of adapted solution (or M-solution) under non-Lipschitz condition. As shown in [9] or [10], the following two functions satisfy the assumption of $\rho$ in (H2),

$$
\begin{gathered}
\rho_{1}(x)=\left\{\begin{array}{cc}
x \ln \left(x^{-1}\right), & x \in[0, \delta], \\
\delta \ln \left(\delta^{-1}\right)+\dot{\rho_{1}}(\delta-)(x-\delta), & x>\delta,
\end{array}\right. \\
\rho_{2}(x)=\left\{\begin{array}{cc}
x \ln \left(x^{-1}\right) \ln \ln \left(x^{-1}\right), & x \in[0, \delta], \\
\delta \ln \left(\delta^{-1}\right) \ln \ln \left(\delta^{-1}\right)+\dot{\rho_{2}}(\delta-)(x-\delta), & x>\delta,
\end{array}\right.
\end{gathered}
$$

with $\delta \in(0,1)$ being sufficiently small. However, the explicit form of $\rho_{i}$ is not easy to get, so now we will give another example to avoid this problem. 
Let us consider the following equation

$Y(t)=\psi(t)+\int_{t}^{T} L(t, s)[f(|Y(s)|)+|Z(t, s)|+|Z(s, t)|] d s-\int_{t}^{T} Z(t, s) d W(s)$,

where $f: \mathbb{R} \rightarrow[0, \infty)$ is defined by

$$
f(x)=\left\{\begin{array}{cc}
0, & x=0, \\
|x|\left[\ln \left(1+|x|^{-1}\right)\right]^{\frac{1}{2}}, & 0<|x|<\delta, \\
\delta\left[\ln \left(1+|\delta|^{-1}\right)\right]^{\frac{1}{2}}, & |x| \geq \delta,
\end{array}\right.
$$

$L(t, s)$ satisfies $\sup _{t \in[0, T]} \int_{t}^{T} L^{q}(t, s) d s<\infty$, where $q>2$ is a constant. It can be shown that $|f(y)-f(\bar{y})| \leq \rho\left(|y-\bar{y}|^{2}\right)^{\frac{1}{2}}$ where $\rho$ can be defined by

$$
\rho(x)=\left\{\begin{array}{cc}
0, & x=0 \\
x \ln \left(1+x^{-1}\right), & 0<x<1 \\
\ln 2, & x \geq 1
\end{array}\right.
$$

We refer the reader to [4] for the proof. Then by Theorem 3.5, it is clear that (4.23) admits a unique M-solution. We can also give the example for adapted solutions in a similar way.

Acknowledgments. The authors would like to thank the anonymous referees for their careful reading of this paper and helpful comments and suggestions.

\section{References}

[1] A. Aman and M. N'Zi, Backward stochastic nonlinear Volterra integral equations with local Lipschitz drift, Probab. Math. Statist. 25 (2005), no. 1, Acta Univ. Wratislav. No. 2784, 105-127.

[2] V. Anh and J. Yong, Backward stochastic Volterra integral equations in Hilbert space, Differential and Difference Equations and Applications, pp. 57-66, Hindawi, New York, 2006.

[3] I. Bihari, A generalization of a lemma of Bellman and its application to uniqueness problems of differential equations, Acta Math. Acad. Sci. Hungar. 7 (1956), 81-94.

[4] A. Constantin, A backward stochastic differential equation with non-Lipschitz coefficients, C. R. Math. Rep. Acad. Sci. Canada 17 (1995), no. 6, 280-282.

[5] N. El Karoui and S. Huang, A general result of existence and uniqueness of backward stochastic differential equation, Backward stochastic differential equations (Paris, 1995996), 27-6, Pitman Res. Notes Math. Ser., 364, Longman, Harlow, 1997.

[6] N. El Karoui, S. Peng, and M. Quenez, Backward stochastic differential equations in finance, Math. Finance. 7 (1997), no. 1, 1-71.

[7] J. Lin, Adapted solution of a backward stochastic nonlinear Volterra integral equation, Stoch. Anal. Appl. 20 (2002), no. 1, 165-183.

[8] J. Ma and J. Yong, Forward-Backward Stochastic Differential Equations and Their Applications, Lecture Notes in Math. Vol. 1702, Springer-Verlag, Berlin, 1999.

[9] X. Mao, Adapted solutions of backward stochastic differential equations with nonLipschitz coefficients, Stochastic Process. Appl. 58 (1995), no. 2, 281-292.

[10] Stochastic Differential Equations and Their Applications, Horwood Series in Mathematics and Applications, Horwood Publishing Limited, Chichester, 1997. 
[11] E. Pardoux and S. Peng, Adapted solution of a backward stochastic differential equation, Systems Control Lett. 14 (1990), no. 1, 55-61.

[12] D. Revuz and M. Yor, Continuous Martingale and Brownian Motion, Springer-Verlag, 2004.

[13] Y. Ren, On solutions of Backward stochastic Volterra integral equations with jumps in hilbert spaces, J. Optim. Theory Appl. 144 (2010), no. 2, 319-333.

[14] T. Wang and Y. Shi, Symmetrical solutions of backward stochastic Volterra integral equations and their applications, Discrete Contin. Dyn. Syst. B. 14 (2010), no. 1, 251274 .

[15] Z. Wang and X. Zhang, Non-Lipschitz backward stochastic volterra type equations with jumps, Stoch. Dyn. 7 (2007), no. 4, 479-496.

[16] _ A class of backward stochastic Volterra integral equations with jumps and applications, preprint.

[17] J. Yong, Backward stochastic Volterra integral equations and some related problems, Stochastic Process. Appl. 116 (2006), no. 5, 779-795.

[18] _ Continuous-time dynamic risk measures by backward stochastic Volterra integral equations, Appl. Anal. 86 (2007), no. 11, 1429-1442.

[19] — Well-posedness and regularity of backward stochastic Volterra integral equation, Probab. Theory Related Fields 142 (2008), no. 1-2, 21-77.

[20] J. Yong and Y. Zhou, Stochastic Control, Hamiltonian systems and HJB equations.Applications of Mathematics (New York), 43. Springer-Verlag, New York, 1999.

YUFENG SHI

SCHOOL OF Mathematics

SHANDONG UNIVERSITY

JINAN 250100, P. R. CHINA

E-mail address: yfshi@sdu.edu.cn

TIANXIAO WANG

SCHOOL OF MATHEMATICS

SHANDONG UNIVERSITY

JinAN 250100, P. R. CHINA

E-mail address: xiaotian2008001@gmail.com 